\title{
Propuesta vial para atacar el problema de contaminación en la Ciudad de México
}

Vial proposal to tackle the problem of pollution in Mexico City

\author{
José Raúl Luyando Cuevas ${ }^{1}$ \\ Julio Herrera Revuelta ${ }^{2}$
}

El objetivo del trabajo es reflexionar sobre el tema de la contaminación atmosférica desde la problemática vial y proponer políticas para su disminución en la Ciudad de México. Para lo cual, desde la metodología sintética se realiza una propuesta teórica que trata de solucionar desde la perspectiva del costo-beneficio una de las tantas incidencias que genera esta situación. El planteamiento suponemos es novedoso, pues aunque hay artículos y libros que tratan el tema y tocan sus especificidades, no encontramos alguna iniciativa que ataque el problema en los términos en que lo hace esta investigación. El resultado de esta investigación es que el propietario de un vehículo privado en la Ciudad de México tiene que pagar, entre otros, un costo social por utilizar espacios públicos, una situación que no se ha atacado y tiene un impacto cada vez más negativo en el bienestar de su población.

The objective of this work is to reflect on the issue of air pollution since of road problem and propose policies for their reduction in Mexico City. So, since the synthetic method, it is a theoretical proposal that tries to solve from the perspective of cost-benefit one of many incidents generated by this situation. We suppose that the approach is novel because although there are articles and books that deal with the subject and touch its specificities; we did not find any initiative that attacks the problem in the terms in which this research does. The result of this investigation is that the owner of a private vehicle in Mexico City has to pay, among others, a social cost for using public spaces, a situation that has not been attacked and has an increasingly negative impact on the welfare of its population.

\footnotetext{
1 Profesor Tiempo Completo del Instituto de Investigaciones Sociales de la Universidad Autónoma de Nuevo León. Instituição: Universidad Autónoma de Nuevo León. México. E-mail: jrlucu@gmail.com

2 Profesor en la Universidad de Valladolid en España. Publicaciones: Distribución funcional de la renta y crecimiento de la demanda: México y España.
} 
Palabras clave: Contaminación, calles y avenidas, tráfico, aparcar, costos.

Keywords: Pollution, streets and avenues, traffic, park, costs.

1. Introducción

La mala calidad del aire en la Ciudad de México dio pie para que el jefe de gobierno de la misma impusiera restricciones a la circulación de autos particulares, en meses en dónde señalaba las condiciones climáticas hacen difícil la dispersión de contaminantes, lo que se denominó programa Hoy No Circula Ampliado. Este programa fue implementado del 5 de abril al 30 de junio del 2016, pues las autoridades de la ciudad indicaron que después de esta última fecha las condiciones atmosféricas cambiarían y habría mejores circunstancias para regresar al esquema que se ha venido implementando de tiempo atrás, denominado programa Hoy No Circula. Este último se implementó en el año de 1989 y su objetivo, en teoría, era sacar de circulación, en días hábiles (de lunes a viernes) a una quinta parte del parque vehicular por día. La decisión sobre que autos dejaban de circular y el día que lo debían de hacer se tomó considerando el número postrimero de la placa que todo auto requiere para transitar. En un principio se planeó sólo utilizarlo en los meses posteriores a la temporada de lluvia, en lo que se considera la temporada de invierno, pero desde el año de 1990 fue implementado para realizarse todo el año. Lo anterior fue asistido por un esquema de verificación vehicular que realizaban los autos dos veces al año, con base en -de nueva cuenta- el último digito de la placa, el cual, debía monitorear una norma de emisión de gases contaminantes para determinar si tenían restricciones para transitar o no y que grado de restricción tenían. Es decir, se determinaba si podían circular o requerían de algún ajuste o reparación, además de que se establecía si podía circular libremente o habría alguna limitación a la circulación, para lo cual, se le otorgaba al dueño un holograma.

Las concesiones y limitaciones en la actualidad, en general, se pueden organizar de la siguiente manera:

a) Exento.- Para vehículos eléctricos e híbridos. Estos no requieren realizar la verificación y, por tanto, no están sujetos al programa Hoy No Circula.

b) Doble Cero.- Concedida a vehículos con hasta dos años de antigüedad. Es un holograma con el cual los autos podrán circular todos los días y tendrá vigencia por dos años, siempre y cuando obtenga resultados satisfactorios en las verificaciones requeridas. 
c) Cero.- Es un holograma con el que se podrá circular todos los días -en caso de que el automóvil cumpla con los límites de contaminación permitidos-, pero que tendrá una vigencia semestral.

d) Uno.- Es un holograma que restringe la circulación de los vehículos. No podrán transitar un día entre semana (de las 05:00 a las 22:00 horas) y dos sábados por mes. Su vigencia es semestral, con base en no sobrepasar los límites máximos de emisión requeridos por las autoridades correspondientes.

e) Dos.- Es otro holograma que restringe aún más la circulación. No podrán transitar un día de la semana (de las 05:00 a las 22:00 horas) y todos los sábados. Su vigencia será semestral cuando obtengan los resultados aprobatorios en los límites máximos de emisión.

En lo referente al programa Hoy No Circula Ampliado, se tienen dos fases: la I, cuando el ozono es mayor a 180 puntos y la ll cuando ozono es mayor a 230 puntos y se suspende cuando es menor o igual a 150 puntos, haciéndose hincapié que para esto último se consideraría también que las condiciones meteorológicas fueran favorables para la dispersión de contaminantes. Al día siguiente de declararse la fase I dejarían de circular vehículos con holograma de verificación 1, 2 y permisos con terminación numérica de placa de circulación non o par de manera alternada de acuerdo a la última declaración de contingencia. Se restringirá la circulación del 50\% del parque vehicular, es decir, dejarán de circular un día los autos con terminación de placas en número par y el siguiente los vehículos cuya placa termine en número non, esto incluye el transporte público federal.

2. Problemas del Transporte Privado 3

Los vehículos son una de las principales fuentes de contaminación atmosférica, principalmente por tres tipos de contaminantes: ozono, monóxido de carbono y dióxido de nitrógeno. En este sentido, se debe considerar que es una fuente de contaminación móvil y no estática, por lo cual, los perjuicios que causa al medio ambiente dependen de la distancia recorrida, la saturación de las avenidas (horas pico u otras), la calidad de los combustibles que utiliza y el estado técnico de la unidad. De entre estas problemáticas, el gobierno de la Ciudad de México ha puesto especial interés en el estado técnico de la unidad, el Gobierno Federal se ha encargado de ver lo concerniente a la

\footnotetext{
${ }^{3}$ Son los servicios de transporte que no son para el público en general. La diferencia con respecto al transporte público se da en diferentes aspectos como: no hay rutas fijas para el transporte privado, los horarios los fija el propietario o quién maneje la unidad a su conveniencia y la velocidad es una elección del propietario o quién maneje la unidad -restringido por lo que se supone es el reglamento vial del lugar y la infraestructura.
} 
calidad de la gasolina y entre ambos han puesto, en algún punto en el tiempo, atención a la distancia recorrida ${ }^{4}$. Pero pensamos que se ha dejado de lado o se ha puesto poca atención a lo que se denomina saturación de la vía de tránsito vehicular, entendida esta última como: "Espacio físico destinado exclusivamente al tránsito de vehículos"5. En el caso específico del transporte privado, se debe señalar que el concepto de saturación que utilizamos es para indicar que el número de vehículos de este tipo ha excediendo la capacidad que tienen calles y avenidas en la Ciudad de México ${ }^{6}$, es decir, en el sentido de que el propósito primordial de cualquier vía de transito vehicular es precisamente permitir el desplazamiento o circulación eficiente de automóviles. Un dato que nos podría ayudar a visualizar esta problemática es comparar el número de habitantes con el de vehículos en la Ciudad de México.

\section{[Gráfica 1]}

En la gráfica 1, se puede observar que la población total disminuyó entre 1980 y 19907. Desde 1990 el tamaño de la población aumentó, para en 2015 regresar a una un poco mayor de la que había en 1980. Por su parte, el crecimiento en el número de automóviles ha tenido tres tipos de evolución, como se puede observar en el gráfico. De 1980 a 1995 el incremento fue moderado; después de 1995 y hasta 2005 se observa una progresión mayor; después de 2005 y hasta $2014^{8}$ existe un acelerado crecimiento en el número de unidades. Y, aunque, la población entre 1980 y 2015 no ha cambiado en mucho (0.99\%), el crecimiento en el número de vehículos ha sido significativo (153.38\%). Para tener una noción más clara del cambio, en proporción se podría decir que en 1980 sólo $21.17 \%$ de la población poseía un automóvil y para 2015 más de la mitad de la población (53.12\%) tenía uno. En este sentido -considerando sólo a la Ciudad de México-, cabe señalar que el crecimiento poblacional no es la causa del aumento en el número de vehículos -algo que en general se menciona en diferentes investigaciones-. De hecho, el anterior fenómeno es más bien propiciado por dos coyunturas en la economía nacional: las facilidades financieras que se han dado para adquirir automóviles nuevos y los menores costos en los usados 9 . En el caso de los nuevos, por la mayor oferta y variedad de marcas en el mercado, aunado esto a las amplias posibilidades de financiamiento que ofrecen las agencias

\footnotetext{
${ }^{4}$ Encuesta origen-destino 2007, Instituto Nacional de Estadística y Geografía (INEGI), México.

5 Artículo 91 de Ley de Transporte y Vialidad del Distrito Federal. Consultado en: http://www.aldf.gob.mx/archivo-18b25984124e5b832406deb1ea65c408.pdf

${ }^{6}$ Vías primarias y secundarias.

${ }^{7}$ Debido, suponemos, principalmente, entre otras cosas, al desastre natural ocurrido en el año de 1985.

${ }^{8}$ Al no encontrar el dato oficial para 2015, utilizo el año 2014 como una aproximación.

${ }^{9}$ Sin menospreciar que hubo también una mejoría en el poder adquisitivo de una parte de la población.
} 
de autos, los bancos comerciales u otros, para su adquisición ${ }^{10}$. Y en los usados, su depreciación dadas las políticas aplicadas con los programas del Hoy No Circula, aseguran precios accesibles para su adquisición a una parte de la población ${ }^{11}$.

Ahora bien, regresando al problema del crecimiento vehicular, podemos hacer el siguiente ejercicio: suponemos que en promedio un auto mide de largo 4 metros y según las estadísticas de INEGI existían 4,737,749 vehículos en 2015, la longitud en conjunto de todos ellos en metros sería de 18,950,996 y transformados a kilómetros tendríamos 18,951. Considerando que la Secretaría de Movilidad del Gobierno de la Ciudad de México ${ }^{12}$ señala en su página que la longitud total de la red vial es de 10,200 km, esto quiere decir que en longitud una línea formada por todos estos vehículos es mayor en longitud a la red vial de la Ciudad de México - faltaría considerar otros factores como el ancho de las vialidades o el de los autos para tener una visión completa del conflicto, aunque esta primera magnitud nos da una buena perspectiva de la contrariedad-. Es decir, se han dado las condiciones para un aumento masivo en la adquisición de autos, pero no existe una estrategia de incentivos planificada que asegure, por otro lado, la salida de un porcentaje alto de los que ya no son funcionales para el sistema vial dadas las necesidades medioambientales y, por tanto, de movilidad en la región actualmente ${ }^{13}$. Ni el aliciente de una oferta de un transporte público eficiente y cómodo.

En este sentido, suponemos que existe un límite en el sistema, una cantidad máxima de autos que asegura, en alguna medida, la eficiencia circulatoria sobre la vía de tránsito vehicular ${ }^{14}$. Y en la medida en que esto se pueda llevar a cabo, se pueden atacar problemas de contaminación aérea y

\footnotetext{
10 Por ejemplo, como lo señala Sánchez (4 de enero de 2017): “Al cierre de 2016 se registró la venta de un millón 603 mil 672 automóviles, la cifra más alta en la historia para el mercado mexicano y un incremento de 18.6 por ciento comparado con lo registrado un año antes". Periódico El Financiero: Venta de autos en México rompe récord en 2016. México.

${ }^{11}$ Además, se debe considerar la importación que se realiza de autos usados desde E.E.U.U.

${ }^{12}$ Consultado en la página: http://data.semovi.cdmx.gob.mx/wb/stv/estadisticas.html

${ }^{13}$ Si tomamos una medida de carga simple: la longitud total de la red vial entre el tamaño promedio de un auto, tendríamos en metros (10,200,000/4). Lo cual, nos daría soporte para 2,550,000 autos particulares. Es sólo una aproximación pues faltaría tomar en cuenta factores como: el ancho de las calles y avenidas, los otros vehículos existentes (muchos de ellos con longitudes mayores), obstrucción de las avenidas, condiciones en que se encuentra la infraestructura vial, accesibilidad, etc.

${ }^{14}$ Pensamos que el Gobierno de la Ciudad de México tiene una buena idea de cuál debiera ser ese límite, como de forma indirecta se demostró en las contingencias ambientales del 2016, donde por momentos sólo circularon alrededor del 50\% de los autos existentes en ese momento. Si tomamos como base el año 2015, serían 2,368,875 aproximadamente y con medida de carga simple 2,550,000. Algo que consideramos cercano a lo que debiera ser, si tomamos en cuenta que la población es similar a la que había en 1980 y, por tanto, se debiera tomar como factor de crecimiento del parque vehicular -a partir de 1980- el aumento en la red vial que ha habido desde entonces.
} 
enfermedades causadas por ésta ${ }^{15}$. Así como el consumo excesivo de energía y los problemas de congestionamiento. Con respecto a esto último, López (2017) señala:

Además, de acuerdo con el índice de Tráfico de Tom Tom (especialistas en servicios de navegación), la Ciudad de México es la ciudad más congestionada del mundo, superando a mega ciudades como Bangkok, Estambul y Río de Janeiro. Los datos generados mostraron que tener una correcta gerencia de las vialidades y aumento en las opciones de transporte, los tiempos de traslado podrían mejorarse hasta en un 59\%; es decir, con las políticas correctas los ciudadanos podríamos llegar a tener nuestros destinos diarios en menos de la mitad del tiempo.

Ahora bien, como lo señala López (2017), de las dos consideraciones en la correcta gerencia, el actual gobierno de la Ciudad de México ha puesto su atención -sin que esto signifique eficacia-, en el aumento en las opciones de transporte y ha mostrado desdén, en el que consideramos uno de los elementos principales en la correcta gerencia de las vialidades, específicamente: la saturación de la vía de tránsito vehicular. La cual contribuye de manera importante al congestionamiento que diariamente se da en las calles y avenidas de la ciudad, propiciando junto con otros elementos -en determinados momentos-, la contaminación atmosférica y cuando pasa ciertos niveles la contingencia ambiental. Como bien los señala Navarro (20 julio 2014):

En México los automóviles son la principal fuente de contaminación que contribuye a la formación de ozono troposférico, asociado con graves enfermedades cardiopulmonares, por lo que el gobierno no puede seguir retrasando la actualización de las normas que regulan las emisiones a escala nacional.

3. Propuesta

Desde el Gobierno Federal, se contemplan dos estrategias que en algún sentido atacan el problema de la contaminación -que cabe decir han tenido buenos resultados en otros países-: aumentar el precio de la gasolina y diésel (gráfica 2) y aplicar la Norma Oficial Mexicana (NOM-041SEMARNAT-2015 ${ }^{16}$ ), que regula los límites máximos de emisión de gases contaminantes, provenientes del escape de los vehículos que usan gasolina como combustible. En el primer caso, se buscaría hacer costoso el uso del auto a los ciudadanos y así contrarrestar la contaminación que generan. Aunque, más que la preocupación por esto último, en su momento se habló más de eliminar

\footnotetext{
15 “La contaminación del aire representa un importante riesgo medioambiental para la salud. Mediante la disminución de los niveles de contaminación del aire los países pueden reducir la carga de morbilidad derivada de accidentes cerebrovasculares, cánceres de pulmón y neumopatías crónicas y agudas, entre ellas el asma. Cuanto más bajos sean los niveles de contaminación del aire mejor será la salud cardiovascular y respiratoria de la población, tanto a largo como a corto plazo". Organización Mundial de la Salud, consultado en: http://www.who.int/topics/air_pollution/es/

${ }^{16}$ Diario Oficial de la Federación (10 junio 2015). "Norma Oficial Mexicana NOM-041-SEMARNAT-2015"
} 
el subsidio que el Gobierno federal otorgaba a este producto y los aumentos que se daban por un impuesto indirecto en este producto que finalmente pagan los consumidores, denominado Impuesto Especial sobre Producción y Servicios (IEPS) y que no necesariamente sirve para combatir el problema, sino para realizar una recaudación mayor dado que la demanda de gasolina es inelástica, como bien lo señaló Patiño (28 diciembre 2016), en una cita de su reportaje:

No existe un mecanismo para asegurar que estos ingresos se destinen para impulsar mejoras en el medio ambiente, refirió Limón. Lo que se anuncia no debilita la situación financiera del gobierno Federal... Nos permite tener también una fuente eficiente y estable de recaudación.

\section{[Gráfica 2]}

En lo referente a la NOM, es un requisito que en otros países se aplica directamente al productor de autos, pues el número de agentes que tienen que ser regulados es menor y así el gobierno se asegura que cada unidad vendida en su territorio cumple con el requisito de una emisión máxima de gases nocivos. Pero en el caso de México y específicamente a la Ciudad de México, esto se aplica tanto a autos nuevos como usados, por tanto, se podría ver como una situación en dos niveles: la de autos nuevos regulada por el Gobierno federal y la de autos usados, principalmente por el Gobierno local aunque también interviene el federal. En el caso de los autos nuevos se podría hablar, por lo menos, de un conflicto de intereses, pues al mismo tiempo que se tiene que imponer la norma, se invoca la necesidad de esta clase de inversión para generar fuentes de empleo e ingresos en el país, por tanto, la pregunta en este sentido es si un Gobierno con la urgencia de solicitar inversión -una posición de debilidad- está en condiciones de regular a un sector tan poderoso y con una posición fuerte. Como lo señala Xantomitla (27 mayo 2017):

Refirió que México fue atractivo como socio comercial para Estados Unidos, entre otras cosas, por la laxitud de la legislación ambiental que aseguraba a las empresas que no iban a ser molestadas con estándares altos de protección al medio ambiente. $Y$ en estos años constatamos que no ha mejorado sensiblemente.

Además de lo anterior, se debe considerar que el país se ubica en Transparency International como uno entre los que existen altos niveles de corrupción ${ }^{17}$ y ello también podría estar afectando la supervisión estricta de la norma.

\footnotetext{
${ }^{17}$ Esta Organización ubica a México en 2016 en el Rank 123 y un score de 30 y señala que: "Los países con menor rango en el índice están plagados de instituciones públicas poco fiables y con un mal funcionamiento, como la policía y el poder judicial. Incluso cuando las leyes anti-corrupción están en la Constitución, en la práctica son a menudo saltadas o ignoradas. Las personas enfrentan frecuentemente situaciones de soborno y
} 
En el caso de los autos usados, existe el programa denominado Hoy No Circula que pensamos tiene dos vertientes: la principal, es la apuesta por una mejoría técnica-mecánica en el transporte privado de pasajeros para atacar el problema de la contaminación atmosférica. En este sentido, es evidente la postura del programa al exentar de restricciones a la circulación a los autos que utilizan energía eléctrica ${ }^{18}$ para operar. Además, el holograma doble cero (para circular diariamente) sólo lo pueden obtener automóviles con motores de combustión interna que utilizan gasolina o diésel que tengan dos años de antigüedad y, por tanto, la tecnología que en ese momento se supone es la más moderna y eficiente. La segunda, pensamos, menos ostensible y tangible, sería la reducción en el uso de este medio de transporte, poniendo acento en mejorar el mantenimiento de aquellos que no cumplen con las condiciones mecánicas que impone el programa, es decir, que no observan la norma al momento de realizar su verificación, la cual, como se señaló, deben realizar dos veces al año la mayoría de los automóviles en la Ciudad de México; en el supuesto de que si no la pasan y el mantenimiento o reparación requeridos son muy costosos (además de sumar las posibles multas o el tiempo utilizado por el propietario en tramites), los dueños podrían sacar de circulación esas unidades. Un supuesto que dadas las carencias y deficiencias del transporte de pasajeros público masivo o los precios del individual y la comodidad que en este sentido significa un transporte particular, será difícil que se cumpla en altos porcentajes. Aún más, si consideramos lo señalado anteriormente, en el sentido de la depreciación que han tenido los autos con hologramas mayores al cero; algunos de los dueños, en la condición antes descrita, aún los podrán vender a otros estados de la República mexicana que tengan normas menos estrictas, buscando comprar algún otro en mejores condiciones y precio bajo ${ }^{19}$. Como bien lo señaló Medina (7 abril 2015):

Dado que no hay desincentivos reales en toda la Zona Metropolitana del Valle de México (ZMVM) para reducir el uso de los automóviles de todo tipo (nuevos o usados), ni opciones reales para evitarlo, como un sistema de transporte público metropolitano de calidad, la adquisición de un auto es garantía de uso irrestricto. Así, el incremento del parque vehicular privado en la ZMVM nos

\begin{abstract}
extorsión, dependen de servicios básicos que han sido socavados por la apropiación indebida de fondos y se enfrentan a la indiferencia oficial cuando buscan la compensación o reparación del daño de esas autoridades" [traducción propia].

${ }^{18}$ El automóvil con motor totalmente eléctrico que se denomina cero emisiones y que se publicita como más amigable con el medio ambiente. $Y$ el hibrido que combina un motor de combustión interna y uno o varios motores eléctricos.

${ }^{19}$ Una situación que sucede por la incapacidad del Gobierno Federal para fijar una norma a nivel nacional que homologuen requisitos, de modo que lo antes descrito no pueda suceder y esos autos tengan que salir forzosamente de circulación. Teniendo presente que buena parte de la problemática en este sentido, se está presentando en otras áreas metropolitanas, entre las que destacan las de Guadalajara y Monterrey.
\end{abstract}


habla de cuánto aumenta a su vez el uso del automóvil en la ciudad y las cifras al respecto son alarmantes.

En últimos días, como lo mencionamos antes, el gobierno de la ciudad, también señaló que impulsará el programa "menos cajones, más ciudad (Sañudo, 2014)" para atacar el problema de la contaminación y sus efectos en la salud de la población que la respira ${ }^{20}$. Poniendo énfasis en que desde las nuevas construcciones de viviendas, se apremie a sus nuevos ocupantes a utilizar el transporte público, un proyecto que pensamos debió iniciar con una oferta de un transporte público más eficiente y cómodo, para luego llegar a esta propuesta, pues de otra manera, aunque la vivienda no tenga cajón para auto, los ocupantes buscando comodidad y eficiencia (considerando que tienen los ingresos suficientes) adquirirán una de estas unidades para transportarse, y al no tener alternativa, la estacionaran en la calle -una situación que actualmente se puede apreciar en casi la totalidad de calles y avenidas secundarias de la ciudad, dada la cantidad de autos existente-. De hecho esta situación que parecería estar restringido a la gente de menores ingresos, no lo es, pues es común observar en casas de zonas con altos ingresos que los cajones no les son suficientes (ejemplo: tienen 5 autos y sólo 3 cajones) y ocupan, de igual modo, la calle para estacionar éstos. Es un fenómeno que se ha extendido dado que no hay una relación congruente entre las unidades existentes y el número de cajones de estacionamiento. Al existir tal desproporción, las calles y avenidas se han convertido en aparcamientos, bien para las personas que no tienen uno o para los que tienen más autos que cajones en sus viviendas. La reflexión con respecto a ocupar esos lugares públicos, se puede hacer siguiendo a Hardin (1968), en el sentido de que los automóviles están creciendo de manera acelerada en una superficie finita, lo que significa que la repartición per cápita de los lugares que pueden ocupar ésta disminuyendo. El problema con lo anterior es que se ha puesto poca atención a la congestión generada por los automóviles que se encuentran por cualquier situación inmóviles gran parte del día en las calles de toda la ciudad, un fenómeno que pensamos es similar a la obstrucción que ocasiona el colesterol en las venas del cuerpo humano y que puede obstruir la circulación de la sangre, es decir, un efecto colesterol automovilístico causado por la cantidad de autos que hay en una superficie delimitada.

\footnotetext{
${ }^{20} \mathrm{Y}$ otras acciones como el cobro por circular en algunos puntos de los segundos pisos, ampliación de la capacidad vial y el transporte público, parquímetros y las que se toman cuando se superan los 180 de puntos Imeca, entre las más notorias.
} 
Una situación inicial respecto a lo anterior, es que estamos hablando que esos espacios que ocupan son públicos -pues fueron creados con los impuestos cobrados a los ciudadanos en diferentes épocas- y debieran beneficiar a la población en su conjunto, tanto a la que tiene un automóvil como a la que no. Como bien lo señala Riquelme (31 de julio de 2017):

El hecho de haber adquirido un vehículo automotor, pagar todos los impuestos que de ello derivan y contar con toda la documentación en regla no otorga el derecho de estacionarlo en la vía pública. No existe un derecho a estacionarse, al menos desde el punto de vista de especialistas y organizaciones de la sociedad civil, quienes creen que el espacio público está supeditado al interés común y no sólo al de las personas que tienen automóvil y desean estacionarlo. Aún la calle frente a hogares y negocios es un espacio público y en consecuencia, está condicionado al beneficio de todos.

En este sentido, son ámbitos administrados por el gobierno de la Ciudad de México y lo que se sigue conociendo como Delegaciones (demarcaciones territoriales de la Ciudad de México). Ahora bien, el problema es que al no haber una gestión eficiente de estos espacios, aún y cuando estos gobiernos, en algún sentido, están obligados por ley a hacerlo: en la Ley de Transporte y Vialidad del Distrito Federal ${ }^{21}$, en el artículo 89 del apartado VII de las Vialidades y el Tránsito, en generalidades, se señala: "El retiro de la vialidad de los vehículos y objetos que ilícitamente obstaculicen, limiten o impidan el uso adecuado de la vialidad o pongan en peligro el tránsito de personas o vehículos". La misma ley en sus artículos 8 y 9 también señalan situaciones al respecto. En el caso del primer artículo, la facultad es para Seguridad Pública: "Ill.- Mantener dentro del ámbito de sus atribuciones, que la vialidad esté libre de obstáculos u objetos que impidan, dificulten u obstaculicen el tránsito vehicular y peatonal, excepto en aquellos casos debidamente autorizados" y en el segundo la potestad es para las Delegaciones “II.- Mantener la vialidad libre de obstáculos u objetos que impidan, dificulten u obstaculicen el tránsito vehicular y peatonal, excepto en aquellos casos debidamente autorizados". La situación que plantean estos artículos la llevan a cabo estas autoridades de manera deficiente y errática -más como un dispositivo que genere ingresos al gobierno por multas que una solución a un problema más global-. Además de plantear mecanismos burocráticos a la acción ciudadana y así dificultar aún más una solución acompañada -para que un ciudadano pida el retiro de un vehículo abandonado o mal estacionado, deberá efectuar un trámite ante la Secretaria de

21 Ley de transporte y vialidad del Distrito Federal, Asamblea Legislativa del Distrito Federal. http://www.aldf.gob.mx/archivo-18b25984124e5b832406deb1ea65c408.pdf 
Seguridad Pública y Vialidad ${ }^{22}$ y la respuesta tarda varios días-, recordando nuevamente que es una acción que por ley debieran llevar a cabo los diferentes órdenes de gobierno. Ocasionando que se produzca lo que podemos llamar una utilización individualizada del espacio público, una situación cercana a la planteada en la tragedia de los comunes, donde desde la lógica de la maximización de la utilidad se tendrán dos componentes: uno positivo, pues el individuo se beneficiaría de la utilización de un espacio que es prácticamente gratis ${ }^{23}$ y podría aumentar su utilidad al ocupar más de ellos -en una situación extrema, podría apropiarse de esos lugares e imponer sus propias reglas, con lo cual, su utilidad estaría cercana a 1-. El negativo, sería el sobreuso individual de los espacios, una situación que afecta de forma compartida y negativa la utilidad de otros, pero sólo en pequeñas fracciones. De lo cual, se deduce que el actuar racional de todo individuo, ante esta situación, es utilizar tantos espacios como le sean posibles.

La propuesta, por tanto, tiene su raíz en que la propiedad de un vehículo genera externalidades negativas, y entre estas, se encuentra la de ocupar espacios públicos de forma permanente cuando no se tiene un lugar de estacionamiento propio -hay personas que al no contar con estacionamiento en su recinto familiar o tener más autos que lugares donde aparcarlos, se apropian de lugares públicos cercanos a donde residen-. Situación que de entrada vulnera la convivencia social y genera un costo que repercute en el bienestar de la población ${ }^{24}$, pues al ser un bien público, teóricamente se dice que debe ser no rival, es decir, una unidad de ese bien puede ser consumida por más de una persona. En este sentido, la adquisición de un auto bajo estas condiciones genera un costo externo que hasta ahora no ha sido considerado por el gobierno local, por tanto, si

\footnotetext{
${ }^{22}$ Para solicitar el retiro de vehículos de la vía pública, los interesados deberán presentar su requerimiento en las áreas de atención citadas, proporcionando la siguiente información:

1. Nombre del solicitante.

2. Domicilio del solicitante (calle y número, colonia, delegación y Código Postal).

3. Teléfono y/o correo electrónico para aclaraciones y notificaciones.

4. Solicitar el retiro del vehículo mal estacionado o abandonado, así como informar de las placas, modelo, color y marca.

5. Ubicación exacta del vehículo, con referencias (entre que calles se encuentra).

6. Presentar material fotográfico o de video que muestre la obstrucción de la vialidad por algún vehículo. (preferentemente).

${ }^{23}$ Si el individuo paga impuestos, contribuyo con una mínima parte a su construcción o estaría contribuyendo de igual forma a su mantenimiento.

${ }^{24}$ En la Ciudad de México, ante la incapacidad o corrupción de las autoridades, es común que particulares se apropien de lugares en calles y avenidas, con el propósito de sacar usufructo de ellos: alquilándolos, utilizándolos como parte de sus negocios, como extensión de sus viviendas y otros, además de la problemática planteada aquí.
} 
se tuviera en cuenta, suponemos, habría una menor demanda de autos nuevos y otros tantos usados saldrían de circulación al no poder cubrir este otro costo que cabe señalar se sumaría a los otros costos externos que ya existen -señalados anteriormente- para cubrir lo que se pudiera aproximar al costo social (el costo privado más el costo externo) de utilizar un auto en la Ciudad de México. Autores como Hawken, Lovins y Lovins(1999: 42) señalan: “In Tokyo, you can't buy a car without proving that you own or rent a place to park it". Situación que no se ha contemplado, en este caso en la Ciudad de México, y que esta creado problemas a la circulación y, por tanto, mayor contaminación atmosférica ${ }^{25}$. Todo lo anterior, sustentado en el enfoque de que debe de existir un límite en el número de automóviles, el cual, deberá estar determinado por alguna fórmula que contemple entre otras cosas: la extensión del territorio y de vías para la circulación de autos, la población total y la económicamente activa y la cantidad y calidad de los posibles substitutos.

Por tanto, la propuesta se basaría en el costo social para limitar el número de autos, lo anterior se puede estructurar de la siguiente manera (gráfica 3):

\section{[Gráfica 3]}

Donde P es el precio del automóvil y Q la cantidad de ellos. Si sólo se cubre el costo privado, los precios serían bajos y habrá un exceso de demanda de autos Qe. Por otra parte, si se consideran los costos externos para alcanzar el costo social, los precios se elevarían y la demanda disminuiría a una cantidad de equilibrio para el mercado Qn. Ahora bien, si se considera la restricción en el número de autos, se puede arribar a tres situaciones: Primera, dónde Qn coincida con la cantidad de autos que puede soportar la región, dadas las variables antes señaladas. En términos teóricos esta sería la cantidad que equilibraría el mercado, siempre y cuando, se pueda determinar con exactitud su costo externo y el costo privado, este último dado por el libre juego de la oferta y la demanda. Si lo anterior es posible, encontraríamos a su vez la cantidad exacta de autos. El problema con lo anterior, es que hay una gran cantidad de elementos a considerar para encontrar el costo externo, muchos de los cuales son difíciles de medir en términos monetarios, por lo que, en última instancia, cuando hay que medirlos para efectos prácticos se usan aproximaciones, lo que hace difícil obtener el dato con precisión. Por otra parte, también parece complejo observar un mecanismo nítido y sin interferencias

\footnotetext{
${ }^{25}$ La situación en las calles de la ciudad son evidentes: carros aparcados a la izquierda y derecha convierten una calle de 4 carriles en una de dos, de tres en una. En colonias como la Ramos Millán en la Delegación Iztacalco, un auto aparcado en una de sus calles puede obstruir la circulación total en la misma (algo que ocurre en muchos lugares de la ciudad). Y otros problemas similares que operan en la ciudad principalmente por la gran cantidad de autos existentes.
} 
entre oferta y demanda en mercados donde hay condiciones para no competir en precios. Por tanto, dado que el costo social no es exacto, éste puede ser diferente a la cantidad de autos encontrada mediante formula. Segunda, la cantidad de autos encontrada (línea roja obscura) es menor a la obtenida mediante la aproximación dada por el costo social (Qn). Si el gobierno confía en la cantidad obtenida mediante formula -dado que pueden ser variables un poco más fáciles de medir-, deberán aumentar algún otro costo que se sume al costo externo, de tal manera, que el costo social aumente hasta el punto donde coincida con la restricción (costo social aumentado). Tercera, una situación contraria a la planteada anteriormente, la cantidad de autos encontrada (línea roja) es mayor a la obtenida mediante el costo social (Qn). De nueva cuenta, si el gobierno confía en el indicador, pueden rebajar alguno de los factores que afectan el costo externo o rebajar todos los factores en un porcentaje menor, de tal manera que la cantidad alcance Qn (costo social restringido).

En este sentido, el aumento en el costo externo por ocupar lugares públicos nos acercaría un poco más a lo que debiera ser el costo social real y a la cantidad de autos que debiera haber teóricamente en la región ${ }^{26}$. Mientras el costo social no pueda contemplar todos los costos externos que nos lleven al resultado teórico, el gobierno podrá tener una cierta noción de esa cantidad, si logra de manera adecuada aglutinar a los factores que la afectan en una fórmula. La cantidad teórica en este sentido sería un ideal, pues en la realidad lo que hay son aproximaciones, pero aun así, podemos señalar que entre más cercanas se encuentren estas dos magnitudes estaremos un poco más cercanos a la cantidad correcta, pues se están midiendo de manera diferente.

En este sentido, una de las políticas a implementar en lo inmediato es la que ya se aplica en Tokyo, la cual, señala que no se pueda vender un auto nuevo si el comprador no puede demostrar que cuenta con cajón para estacionarlo. Además de cargar el costo externo de estacionarse en la vía pública a los propietarios que lo hacen diariamente, una situación un poco más complicada de implementar. Pero dado que existe un buen número de ciudadanos que si cumplen con la medida de tener tantos autos como cajones de estacionamiento-, se les convocatoria a indicar su situación y creemos que tendrían incentivos para revelarla dado que sería para exentarlos de cualquier pago, de cualquier modo el gobierno tendría que monitorear esto para tener una certeza. La gente que no

\footnotetext{
${ }^{26}$ Como bien lo señala Bull (2003: 90): "El control de estacionamiento consiste en regular la disponibilidad de sitios de parqueo en distintas zonas de la ciudad, con el fin de disminuir la congestión. Controlar el estacionamiento, sea restringiendo el número de sitios disponibles o cobrando por su uso, ha sido reconocido desde hace tiempo como uno de los elementos más efectivos en cualquier estrategia para reducir el uso del automóvil (Enoch, 2002)".
} 
acuda será en la que las autoridades tendrán que poner mayor atención, pues les tendría que cobrar un monto mensual para tener derecho a estacionar su auto en la calle, uno que bien podría salir de un promedio mínimo del que cobran las pensiones de autos privadas por zonas. En los dos casos para minimizar costos de supervisión el gobierno podría utilizar lo que se denomina una estrategia de amenaza creíble, es decir, no monitorear a toda la población pues sería imposible, pero si aleatoriamente a ciertos elemento de ella y en caso de encontrar que no están cumpliendo o mintieron, castigarlos de manera ejemplar y difundir esto ampliamente en medios de comunicación, de tal manera que los que están haciendo esto reconsideren su actuar, al ser más costosa la sanción impuesta que pagar la mensualidad por la pensión pública. Entre más creíble sea la amenaza, menor gasto se necesitará para el monitoreo, en el óptimo el gasto tendría que ser cero.

La estrategia sólo podrá tener éxito, si el dinero recaudado en este tiempo se utiliza para mejorar el transporte público, pues una de sus intenciones es sacar autos privados de circulación y disminuir en esa medida la congestión vial. Pero las personas que dejen de utilizar el transporte privado necesitarán de un transporte público gradualmente más eficiente en todo sentido y cómodo. Si el gobierno es ineficaz o incompetente para realizar este tipo de proyectos o existe una corrupción tal que mucho de este dinero no se destine al propósito indicado, el proyecto fracasará y se seguirán utilizando remedios cortoplacistas que no solucionan el problema de la contaminación y sus efectos en la salud de los seres vivos que habitan la región, con lo cual, se deja de lado la predica que hace principalmente el gobierno federal en cuanto a que el desarrollo del país y, por tanto, de los estados se debe hacer de forma sostenible.

\section{Discusión}

La congestión por la existencia de un alto número de automóviles, en este caso en la ciudad de México, tiene una problemática que no se ha considerado plenamente y que pensamos es una de las principales causantes de la contaminación atmosférica, además de que generan problemas sociales y también económicos. Es tal la cantidad en la ciudad (además de los que entran en ella de los estados aledaños) que en muchas calles o avenidas de la misma (no únicamente en el centro y zonas lindantes) los autos estacionados están convirtiendo avenidas o calles de cuatro carriles en unas de tres o de dos -en caso de que se puedan estacionar a ambos lados de la acera-. Además, si algún o algunos camiones repartidores de bienes o servicios o algún o algunos autos particulares por cualquier circunstancia necesitan parar en la calle o avenida, en el ejemplo antes señalado, pasan de 
cuatro a un carril. También hay que considerar los casos extremos, en donde un auto estacionado puede obstruir la circulación de calles estrechas enteras, no de gran longitud, pero que problematizan la circulación en una área que de otro modo podría ser más ágil y eficiente ${ }^{27}$. Al mismo tiempo, existen micro-negocios, como los talleres mecánicos y otros denominados de hojalatería y pintura para autos que utilizan la calle como parte de sus locales, apoderándose de esos espacios permanentemente y en caso de aumentar la demanda por sus servicios ocuparán otros más. De la misma manera, bares, restaurants y micro-negocios (diferentes a los antes mencionados), utilizan este espacio público como propio en las horas en que realizan sus actividades y existen muchas calles o manzanas enteras que son privatizadas por vecinos, bajo el pretexto de la inseguridad. Una situación que debiera estar estrictamente supervisada y regulada por las autoridades, tanto Federales como Estatales, pues son actividades que afectan la economía y la buena convivencia ciudadana y, por tanto, el bienestar social. Además que algunas de ellas son altamente contaminantes por el uso que requieren de solventes, lubricantes y gasolinas, desechos estos últimos, que de ir a las alcantarillas estarían causando mayores impactos y costos a la ciudad que el beneficio que pueden generar los empleos que crean. Una situación en la que se debería poner más atención y que ayudaría de alguna manera a lo que se podría denominar las buenas maneras de conducir y acometer desde esta trinchera el problema, es la de otorgar licencias de conducir sólo a aquellos ciudadanos que demuestren ser capaces de hacerlo. Por ejemplo, en España para obtenerla se debe realizar una prueba teórica, una prueba práctica y un test psicotécnico, en cambio en México, no existen obstáculos para que cualquier ciudadano pueda conseguir una, este último caso se puede ver más como un objetivo recaudatorio que uno que busca una buena convivencia vial y bienestar social.

Por su parte, el cobro por ocupar el espacio público como se propone en esta investigación puede tener el inconveniente de que la sociedad perciba que los más perjudicados van a ser los que tienen los menores ingresos y podría haber reticencias. Pero en realidad es todo lo contrario, si los congestionamientos son causados en mayor medida por autos particulares, el transporte público que circula por esas vías también saldrá afectado, causando quebrantos a sus pasajeros (que suponemos son los habitantes con bajos recursos económicos), siendo estos últimos los que en menor medida contribuyen al embotellamiento, pues los microbuses tienen capacidad de albergar alrededor de 30 pasajeros, mientras que los autos en su mayoría trasladan a un sólo ocupante. La liberación de vías

\footnotetext{
${ }^{27}$ Caso que se da en colonias como la Ramos Millán en la Delegación Iztacalco, aunque existen otros muchos ejemplos.
} 
agilizaría el tránsito y podría bajar las tarifas de los transportes públicos, pues utilizarían menos gasolina, podrían ir a mayor velocidad, el desgaste de las unidades sería menor y podrían dar un mayor número de vueltas entre sus bases ${ }^{28}$. Por otra parte, se podrían crear empleos formales, pues como lo señala Bull (2003: 94):

En países con mano de obra de bajo costo o elevados niveles de desempleo, es posible manejar los estacionamientos con cobradores humanos premunidos de aparatos registradores del tiempo estacionado. Así, además de crear fuentes de trabajo formal, se puede cobrar el tiempo exacto de uso, sin perjuicio de que exista un monto mínimo por el hecho de estacionarse.

En el caso de México, la posibilidad de mano de obra barata y elevados niveles de subempleo existen, una situación que se podría utilizar para implantar el sistema antes descrito sin altos costos.

Por último, se debe considerar que la propuesta restringe en alguna medida la demanda de autos nuevos y promueve desde los costos la salida de usados. En el primer caso, creemos que el inconveniente sería menor, teniendo en consideración que se podría afectar el aliento que los últimos gobiernos han dado a la instalación de plantas armadoras de autos en México, pues la mayoría de estos productos finales son exportados a otros países, principalmente a los Estados Unidos de América ${ }^{29}$. Por otra parte, el programa que ha implementado el gobierno de la Ciudad de México (Hoy No Circula) propone y promueve la renovación del parque vehicular, por lo que la demanda interna para este sector de la producción no decaería y por el contrario ha sido estimulada. En lo referente a los autos usados, se supone que saldrían en promedio los que no tienen las condiciones necesarias para poder seguir operando en la ciudad. Es en esta situación donde se vería realmente la eficacia del gobierno para generar alternativas eficientes y cómodas, sin esto último, como cabría intuir, la propuesta podría generar protestas sociales, primando el interés individual o de grupo sobre el bienestar social. Una situación que debe catalizar el gobierno como promotor del interés social, dado que en lo individual, una gran mayoría no tienen la conciencia del daño que se causa al ambiente y en última instancia a la salud de los seres vivos que habitan la región.

\footnotetext{
${ }^{28}$ Las bases se refieren a la ruta de inicio y fin que tienen asignadas y autorizadas estas unidades, por tanto, salen de una para llegar a otra en las horas que dedican los choferes a manejarlas.

29 “México obtuvo cifras históricas de producción y exportación de vehículos en el 2014, con 3 millones 219,786 unidades ensambladas y 2 millones 642,887 de envíos al exterior; carrera en la que se colocó como el séptimo fabricante de autos en el mundo, al desbancar a Brasil, y apuesta a que en el 2015 romperá nuevo récord". Periódico el Economista: Industria bien aceitada acelera y bate nuevo reto, México.
} 


\section{Conclusiones}

La propuesta sobre costos se hace desde una óptica que se ha implementado en muchos países (costo-beneficio), pero enfocándonos en una problemática que se ha generado por el enormemente crecimiento en el número de autos en la Ciudad de México y la falta de una gestión pública adecuada a este respecto. Por tanto, se fundamenta en que la propiedad de un vehículo genera externalidades negativas, y entre estas, se encuentra la de ocupar espacios públicos de forma permanente cuando no se tiene un lugar de estacionamiento propio, lo cual, origina lo que denominamos un efecto colesterol en las vías de comunicación (para el desplazamiento de vehículos). Se señala en este sentido que el número total de automóviles en una región no puede ser ilimitado y una buena idea para acercarnos al número límite de éstos debería considerar la longitud de sus vías de comunicación, la extensión del territorio, la población total y la económicamente activa y la cantidad y calidad de los posibles substitutos ${ }^{30}$ o la de considerar cobrar tanto los costos privados

\footnotetext{
${ }^{30}$ Para el caso de la Ciudad de México, varios estudios señalan que el programa Hoy No Circula, no ha dado los resultados esperados, Bull (2003: 112): "Estudios indican que los impactos negativos de esta medida son mayores que los positivos, entre otras causas, debido a la adquisición de un segundo vehículo, de modo que muchos, en la práctica, terminan no siendo afectados por la restricción. Evidencias indirectas sugieren también que la polución ambiental se ha exacerbado debido a la restricción (Tovar, 1995)".
}

\section{REFERENCIAS BIBLIOGRAFICAS}

ASAMBLEA LEGISLATIVA DEL DISTRITO FEDERAL, IV Legislatura. Ley de Transporte y Vialidad del Distrito Federal. Publicada en la Gaceta Oficial del Distrito Federal el 26 de diciembre de 2002, Disponible en: http://www.aldf.gob.mx/archivo-18b25984124e5b832406deb1ea65c408.pdf. 
BULL, Alberto [Compilador]. Congestión de tránsito: el problema y cómo enfrentarlo. Santiago de Chile, Cuadernos de la CEPAL N 87, 2003.

CIUDAD DE MEXICO. Catálogo único de trámites y servicios. Seguridad Pública y Vialidad. Recuperado el 17 de enero de 2017, de: http://www.tramitesyservicios.df.gob.mx/wb/TyS/retiro de vehiculos abandonados o mal esta $\underline{\text { cionados }}$

CONSEJO NACIONAL DE EVALUACION DE LA POLITICA DE DESARROLLO SOCIAL. Medición de la Pobreza: Anexo Estadístico de Pobreza en México. Recuperado el 9 de abril del 2017, de: http://www.coneval.org.mx/Medicion/MP/Paginas/AE pobreza 2014.aspx

ENOCH, M. P. UK parking cash out experience, and lessons from California. Londres, Traffic Engineering + Control (TEC), vol. 43, № 5, 2002.

GONZALEZ, Lilia. Industria bien aceitada acelera y bate nuevo récord. Ciudad de México Periódico El Economista, 8 de enero del 2015. Disponible en: http://eleconomista.com.mx/industrias/2015/01/08/produccion-autos-mexico-crecio-27diciembre-amia.

HARDIN, Garrett (1968). The Tragedy of the Commons (La tragedia de los comunes, Horacio Bonfil Sánchez, trad.). Ciudad de México, Gaceta Ecológica, núm. 37, Instituto Nacional de Ecología, 1995. Disponible en: http://www.ine.gob.mx/.

HAWKEN, Paul, LOVINS, Amory and LOVINS, Hunter. Natural Capitalism: Creating the Next Industrial Revolution. Boston, Little Brown \& Co, 1999.

INSTITUTO NACIONAL DE ESTADISTICA Y GEOGRAFIA. Sistema Estatal y Municipal de base de datos: vehículos registrados según clase de vehículo, total. Recuperado el 13 de febrero del 2017, de: http://sc.inegi.org.mx/cobdem/index.jsp. 
INSTITUTO NACIONAL DE ESTADISTICA Y GEOGRAFIA. Volumen y crecimiento: población total por entidad federativa, 1895 a 2010. Recuperado el 17 de enero de 2017, de: http://www3.inegi.org.mx/sistemas/sisept/Default.aspx?t=mdemo148\&s=est\&c=29192.

INSTITUTO NACIONAL DE ESTADISTICA Y GEOGRAFIA. Cuéntame (dato de población total para el año 2015). Recuperado el 17 de enero de 2017, de: http://cuentame.inegi.org.mx/monografias/informacion/df/default.aspx?tema=me\&e=09.

INSTITUTO NACIONAL DE ESTADISTICA Y GEOGRAFIA. Encuesta origen-destino 2007 (cabe señalar que se realizó otra en 2017). México. Recuperado el 17 de mayo de 2017, de: http://www.beta.inegi.org.mx/contenidos/programas/eod/2017/doc/conceptual eod 2017.pdf

LOPEZ, Gladis. Movilidad eficiente, crecimiento sostenible. Agencia Quadratín, 2017. Disponible en: https://www.quadratin.com.mx/opinion/movilidad-eficiente-crecimiento-sosteniblegladislopez-blanco/. MEDINA, Salvador. El alarmante crecimiento de autos. NEXOS: la burbuja el blog de la metrópoli, 7 de abril 2015. Disponible en: $\underline{\text { http://labrujula.nexos.com.mx/?p=305 }}$

MENDEZ, César. ¿Cuánto ha subido el precio de la gasolina en México en 6 años? ComparaGuru.com Compara y Ahorra, 4 enero 2017, Disponible en: https://www.comparaguru.com/blog/cuanto-hasubido-el-precio-de-la-gasolina-en-mexico-en-6-anos

NAVARRO, Israel. Autos, primera fuente de contaminación en el país. Ciudad de México, Milenio.com, sección Región, 20 julio 2014, Disponible en: http://www.milenio.com/region/Autosprimera-fuente-contaminacion-pais-Mexico-enfermedades-humoozono troposferico 0 338966122.html ORGANIZACION MUNDIAL DE LA SALUD (OMS). Temas de Salud: Contaminación del Aire. Recuperado el 27 de marzo de 2017 de: http://www.who.int/topics/air pollution/es/ 
PATIÑO, Dainzú. Hacienda recibirá 40\% más IEPS con el gasolinazo. Ciudad de México, El Financiero, sección Economía, 28 diciembre 2016, Disponible en: http://www.elfinanciero.com.mx/economia/hacienda-recibira-40-mas-ieps-con-el-gasolinazo.html RIQUELME, Rodrigo. ¿Existe el derecho a estacionarse? Ciudad de México. Periódico El Economista, 31 de julio de 2017, Disponible en: http://eleconomista.com.mx/distritofederal/2017/07/31/existe-derecho-estacionarse

SANCHEZ, Axel. Venta de autos en México rompe récord en 2016. Ciudad de México, Periódico EI Financiero, Sección Empresas, 4 de enero de 2017, Disponible en: http://www.elfinanciero.com.mx/empresas/venta-de-autos-en-mexico-rompe-record-en-2016colocan-millones.html.

SAÑUDO, Andrés [coordinador]. Menos cajones, más ciudad: el estacionamiento en la Ciudad de México. Ciudad de México, Instituto de Políticas para el Transporte y el Desarrollo México, en el marco del proyecto crecimiento bajo en carbono para las ciudades mexicanas mediante el Desarrollo Orientado al Transporte (DOT), 2014. Recuperado el 14 de febrero de 2017, en: http://mexico.itdp.org/wp-content/uploads/Menos-cajones-m\%C3\%A1s-ciudad.pdf SECRETARIA DE GOBERNACION. Norma Oficial Mexicana NOM-041-SEMARNAT-2015 (10/06/2015), Diario Oficial de la Federación. México, Disponible en: http://dof.gob.mx/nota detalle.php?codigo=5396063\&fecha=10/06/2015 SECRETARIA DE MOVILIDAD (SEMOVI). Vialidades: Estadísticas. Ciudad de México Recuperado el 15 de Mayo del 2017, de: http://data.semovi.cdmx.gob.mx/wb/stv/estadisticas.html.

TOVAR, Ramiro. Mobile source pollution in Mexico City and market-based alternatives, Regulation. The Cato Review of Business and Government, vol. 18, № 2.Washington, D.C., Cato Institute, 1995. Transparency International. Corruption Perceptions Index 2016. The global coalition against corruption. Berlin. Recuperado el 25 de enero 2017, de: https://www.transparency.org/news/feature/corruption perceptions index 2016 
como todos aquellos que se aglutinan en los sociales -o algo que se aproxime lo más posible a ellos-. Es una proposición que ataca un flanco en el que no se había puesto la atención requerida y sus consecuencias en la convivencia diaria y el bienestar social, además de la manifiesta problemática ambiental (calentamiento global, altos niveles de contaminación atmosférica y enfermedades). En esto último hay que recalcar que el actual gobierno de la ciudad ha tenido mucho que ver, por la incapacidad o indolencia que ha mostrado para regular y supervisar problemáticas que están en su jurisdicción y obligación.

Sin duda las propuestas que buscan restringir algo desde el costo, en general, no son bienvenidas, pero en este caso estamos tocando una problemática que está afectando la salud de los seres vivos y en ese sentido se puede ver como una acción superior.

NOTAS

Trabalho enviado em 04 de setembro de 2018

Aceito em 01 de fevereiro de 2019

XANTOMILA, Jessica. EL TLC ha permitido el daño de transnacionales al ambiente. Ciudad de México, La Jornada en línea, sección Sociedad, 27 de mayo de 2017, p.29. Disponible en: http://www.jornada.unam.mx/2017/05/27/sociedad/029n1soc 